\title{
Crack Free GaInN/AlInN Multiple Quantum Wells Grown on GaN with Strong Intersubband Absorption at $1.55 \mu \mathrm{m}$
}

\author{
G. CYWiński ${ }^{a, *}$, C. SkierbiszeWski ${ }^{a, b}$, \\ A. Feduniewicz-ŻMuda ${ }^{b}$, M. SiekACZ ${ }^{a}$, L. Nevou $^{c}$, \\ L. Doyennette ${ }^{c}$, F.H. Julien ${ }^{c}$, P. Prystawko ${ }^{a}$, M. Kryśko $^{a}$, \\ S. Grzanka ${ }^{a}$, I. Grzegory ${ }^{a}$ And S. Porowski ${ }^{a}$ \\ ${ }^{a}$ Institute of High Pressure Physics, Polish Academy of Sciences \\ Sokołowska 29, 01-142 Warsaw, Poland \\ ${ }^{b}$ TopGaN Ltd., 01-142 Warsaw, Poland \\ ${ }^{c}$ Action OptoGaN, Institut d'Electronique Fondamentale \\ Université Paris-Sud, UMR 8622 CNRS, 91405 Orsay Cedex, France \\ Crack free GaInN/AlInN multiple quantum wells were grown by rf \\ plasma-assisted molecular beam epitaxy on (0001) GaN/sapphire substrates. \\ The strain-engineering concept was applied to eliminate cracking effect for \\ growth of intersubband structures on GaN. Indium contained ternary com- \\ pounds of barrier and well layers are contrary strained to the substrate mate- \\ rial. A series of crack free GaInN/AlInN intersubband structures on (0001) \\ GaN was fabricated and investigated. The assumed composition and lay- \\ ered structure were confirmed by room temperature photoluminescence and \\ $\mathrm{X}$-ray diffraction measurements. The intersubband measurements were done \\ in multipass waveguide geometry by applying direct intersubband absorption \\ and photoinduced intersubband absorption measurements. The optimized \\ structure design contains forty periods of Si-doped GaInN/AlInN quantum \\ wells and exhibits strong intersubband absorption.
}

PACS numbers: 68.65.Fg, 78.66.--w, 78.67.De, 78.40.Fy, 81.15.Hi

\section{Introduction}

Recently, there has been increasing interest in the intersubband (IS) transition between quantized electron states in the semiconductor quantum well (QW).

\footnotetext{
*corresponding author; e-mail: gc@unipress.waw.pl
} 
IS transitions have high significance not only for semiconductor physics research but also for their application to ultrafast optoelectronic devices. Unipolar devices relying on IS transitions open new perspective for ultrafast fiber optoelectronic, where using them a significant increase in the bit rate in a single fiber could be achieved. However, because of barrier-well conduction band discontinuity, which determines the shortest wavelength operation limit, materials based on arsenides or phosphides are not suitable for this purpose. New materials with a higher conduction band discontinuity are required. One of the candidates is nitride system, where intensive research works brought to successful fabrication by plasma-assisted molecular beam epitaxy (PAMBE) GaN/AIN IS structures, which absorb light at the telecommunication wavelength region [1-7].

Nitride based IS structures are intensively studied not only because of their application perspectives. They are also interesting in sense of low-dimensional semiconductor physics. In nitrides, due to enhanced electron-phonon interaction in these highly ionic materials, IS absorption recovery times are extremely short, it means that they are typically of the order of $150-400 \mathrm{fs}[4,8,9]$. In nitrides, one can find a few possibilities to fabricate very deep quantum well, where electron band-gap discontinuity is more than $1 \mathrm{eV}$. However, the main stream of research is focused on GaN/AlN system, where lattice mismatch is $\approx 2.5 \%$. This mismatch leads to certain technological problems. Technological difficulties for an epitaxial growth of this system are mainly related to the lack of appropriate substrates. The AlN - barrier material - is tensile strained to GaN. The growth of GaN/AlN device structures on GaN substrate is practically excluded because of cracking problem, which occurs in case of many repetition structures. The only way is to use AlN or AlGaN (with high aluminum content) based substrates, whose quality unfortunately is not satisfied yet. On the other hand, one can propose other technical realization of IS structures based on nitrides, which could use the best available nitride substrate - GaN. The simplest system is GaN/AlInN, where indium content is $\approx 18 \%$ when AlInN barrier is lattice matched to GaN. However, technologically this interesting material is not sufficiently developed up to now. The realization of lattice matched AlInN has been reported only for metal-organic vapor phase epitaxy (MOVPE) technique [10]. Another possibility is to use strain engineering of barrier-well materials to avoid the crack problem, which is present in the case of a GaN/AlN growth of IS structures on GaN. Adding indium to both barrier and well materials one can expect the considerable reduction of average strain between GaInN/AlInN multiple quantum well (MQW) and GaN substrate.

In this communication, we demonstrate the abilities of crack free growth of multiple quantum wells on GaN substrate using as barrier and well materials ternary alloys, which contain indium. Such IS structures grown on GaN substrate contain AlInN and GaInN layers, which are contrary strained to the substrate material. Our GaInN/AlInN IS structures were optimized in sense of growth and optical parameters. We fabricate high quality IS samples, which contain forty 
periods of deep QWs on GaN substrates. Such samples are fully crack free and exhibit $\approx 50 \%$ IS absorption in a multipass waveguide geometry experiment.

\section{Experiment}

We have grown a set of AlInN/GaInN MQWs structures by PAMBE on (0001) $\mathrm{GaN} / \mathrm{Al}_{2} \mathrm{O}_{3}$ templates. The structure scheme of our samples is shown in Fig. 1. They contain a $20 \mathrm{~nm}$ GaInN buffer layer, 40-period AlInN/GaInN MQWs with quantum well thickness in the range of $1.3-1.8 \mathrm{~nm}$, and a barrier thickness of $3 \mathrm{~nm}$, followed by a $100 \mathrm{~nm}$ GaInN cap layer. All GaInN layers have the same indium content. In addition, reference GaN/AlN MQW structures were grown on $\mathrm{Al}_{0.35} \mathrm{Ga}_{0.65} \mathrm{~N}$ templates, where AlInN barriers and GaInN wells were replaced by AlN and GaN, respectively. However, in case of GaN/AlN growth on $\mathrm{Al}_{0.35} \mathrm{Ga}_{0.65} \mathrm{~N}$ substrates, we made only twenty period structures, because this was our technological limit to obtain almost crack free sample. The PAMBE growth for all samples was performed in a VG90 Oxford Instruments MBE system. An ultrahigh-purity gas-handling manifold, together with a Veeco UNI-Bulb rf-plasma cell, was used as a source of activated nitrogen species. The nitrogen source was operating at a power of $240 \mathrm{~W}$, and $0.8 \mathrm{SCCM}$ (denotes cubic centimeter per minute at standard temperature and pressure (STP)) flow of $\mathrm{N}_{2}$ was used. The PAMBE system was pumped by a closed-cycle helium cryopump and has a base pressure of $\approx 10^{-11}$ Torr. Samples were grown without any interruption in metal-rich conditions. The growth rate for all samples was $\approx 0.8 \AA / \mathrm{s}$. The growth temperature was monitored by a thermocouple located behind the substrate holder and by an infrared pyrometer. The growth temperature was about $600^{\circ} \mathrm{C}$.

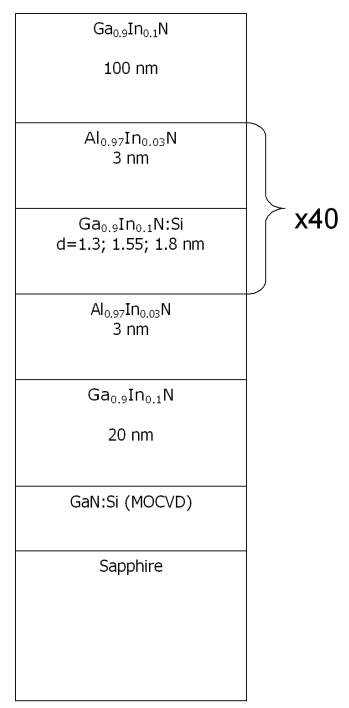

Fig. 1. The layer structure of AlInN/GaInN MQW samples. 
Basing on our previous experience in the PAMBE growth of GaInN/AlInN IS structures [11], where we reported for the first time the growth of such structures on GaN substrates, we have continued our studies. We have increased the Si-doping level in QW regions, which have been insufficient in the previous sample series and we made fine-tuning of growth parameters, which was necessary to obtain the increase in QW repetition number. Our experiments were aiming at a gain in IS absorption by increase in the number of QW repetitions and an increase in the absorption efficiency per QW as well. The optimization of growth parameters was done in two steps. First, we made fine-tuning of growth parameters to avoid droplet formation during longer growth time, where even small metal accumulation on the surface can affect a poor surface morphology. In next step, we approximately determined the maximum number of repetition, where we have not observed any cracks or droplets. We find that we can growth reproducibly forty periods of GaInN/AlInN IS structures on GaN substrates. This number of repetitions was twice bigger than in case of GaN/AlN IS grown on $\mathrm{Al}_{0.35} \mathrm{Ga}_{0.65} \mathrm{~N}$ templates, where single cracks can be observed during inspection using the Nomarski contrast microscopy.

The IS samples have been characterized by X-ray diffractometry (XRD) and room temperature photoluminescence (PL) measurements to confirm the assumed growth structure (see scheme in Fig. 1). The typical XRD data of GaInN/AlInN IS structure is shown in Fig. 2. For these experiments, we used a Philips X'Pert-MPD high-resolution diffractometer. To monochromize the beam, we used a four-bounce 220 Ge reflection (Bartel's monochromator), and as analyzer, we used a two-bounce U-shaped Ge crystal. All measurements were performed in the vicinity of the 0002 reflection. The performed simulations done for XRD data confirm supposed layer compositions and period of MQWs.

The photoluminescence measurements were done using excitation by $244 \mathrm{~nm}$ line of a frequency-doubled continuous wave $(\mathrm{CW}) \mathrm{Ar}^{+}$laser. Two different sample geometries were used. The first - normal-sample geometry - at normal incidence the laser excitation and collection of the PL signal, and second - edge-sample geometry, where laser was focused on the edge of the sample and collection of the PL signal was also from the edge of the sample. The typical PL data and schemes of sample geometries are shown in Fig. 3. In case of the normal-sample geometry we have observed only signal from the MOVPE GaN buffer and no evidence of signal from QW structure. Only in the edge-sample geometry we were able to collect the signal from the QW structure, which could be clarified by presence of $100 \mathrm{~nm}$ GaInN cap layer in our GaInN/AlInN IS samples.

In Fig. 4 there is shown absorption experiment. The optimized set of samples uniformly Si-doped in the QW region at concentration $1 \times 10^{20} \mathrm{~cm}^{-3}$ and with the QWs width $1.3,1.55$, and $1.8 \mathrm{~nm}$ was investigated. All samples in this set have strong absorption in the 1.3-1.55 $\mu \mathrm{m}$ range. Samples were prepared in a way to form a multipass waveguide (the substrate backside and facets at $45^{\circ}$ were 


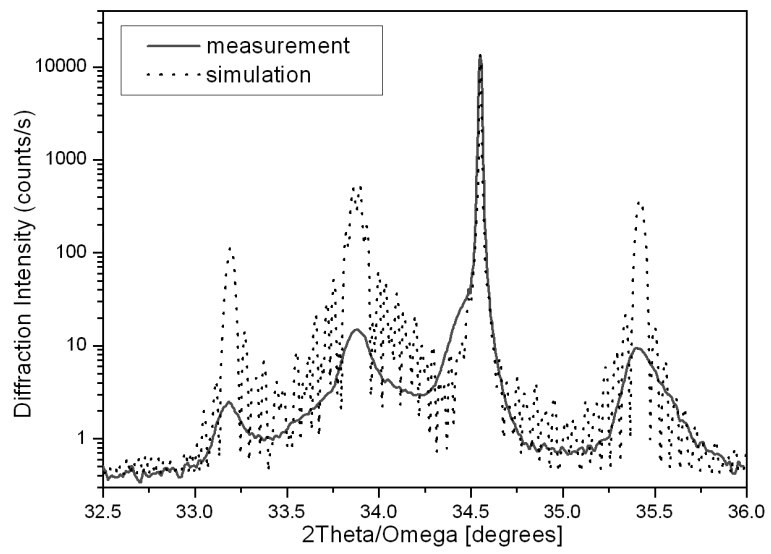

Fig. 2. Result of XRD measurements made on $\mathrm{Al}_{0.93} \mathrm{In}_{0.07} \mathrm{~N} / \mathrm{Ga}_{0.9} \mathrm{In}_{0.1} \mathrm{~N}$ IS structure (continuous line) and numerical simulation (dotted line), which confirms the assumed structure. The nominal thickness of the QW is $1.8 \mathrm{~nm}$.

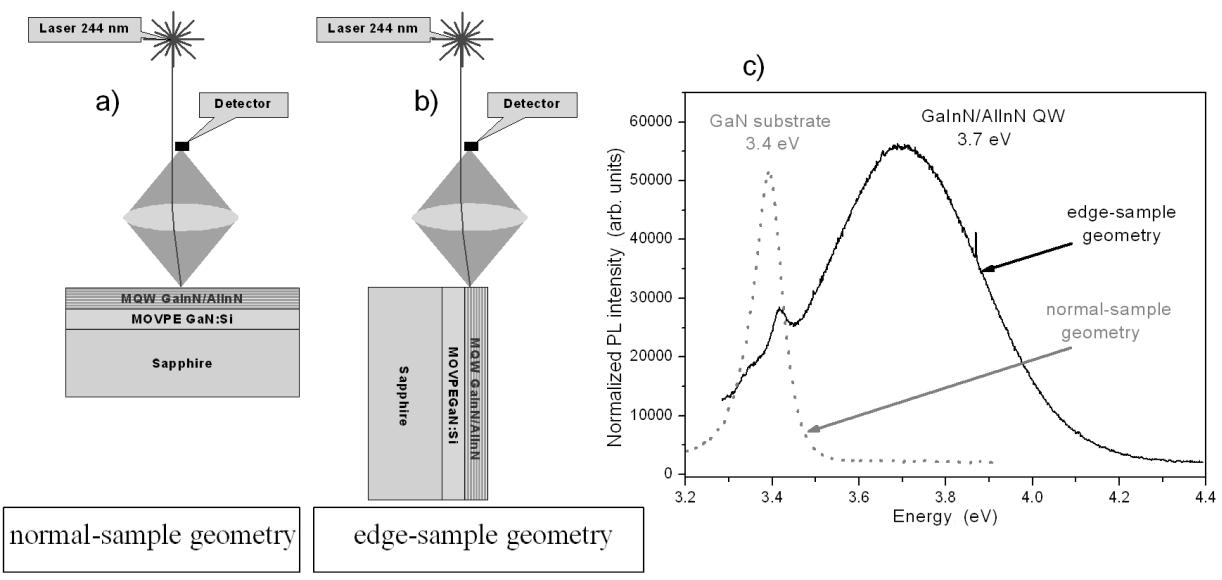

Fig. 3. Room temperature PL measurements for the $1.8 \mathrm{~nm}$ thick QW sample: (a) normal-sample geometry, (b) edge-sample geometry, (c) PL data for both samples geometries.

polished), see Fig. 4a. Due to the polarization selection rules of IS transitions that is, only light with the electric field parallel to the growth axis is absorbed the absorption peaks were obtained from $p$-polarized transmission spectra and no absorption from $s$-polarized light was detected.

Two types of optical experiments were performed on our structures: direct intersubband absorption and photoinduced intersubband absorption (PIA) measurements. In Fig. 4b there are shown the PIA results for our IS sample, which contains forty periods of $1.8 \mathrm{~nm}$ QWs. The advantage of the PIA technique is that we can practically exclude the possibility of another transition, because the PIA 


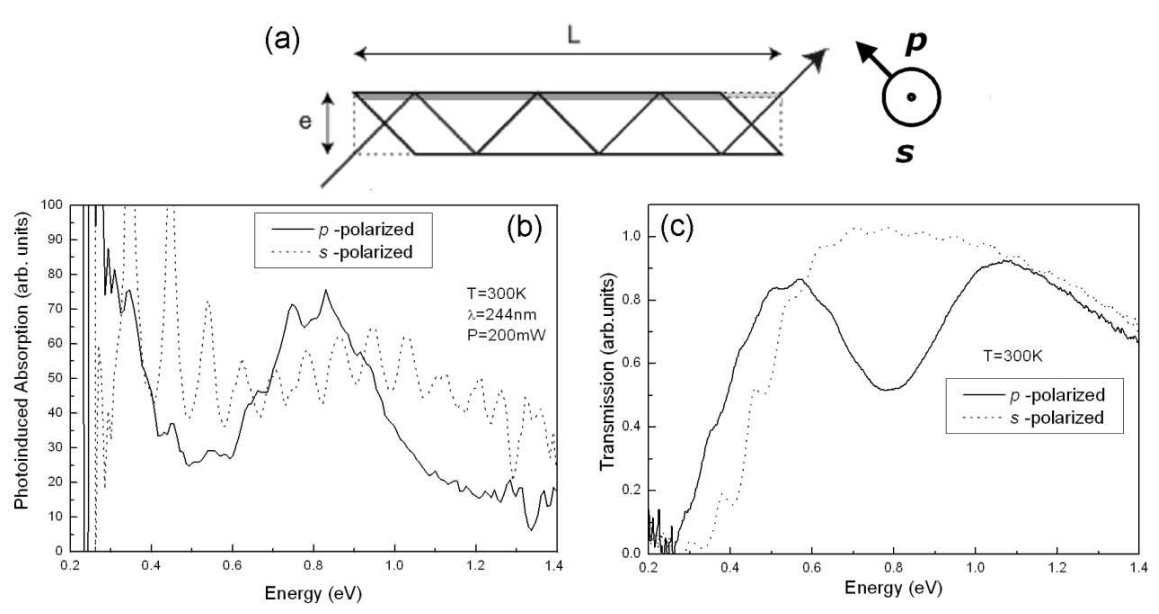

Fig. 4. Room temperature absorption measurements for $p$-polarized (solid line) and $s$-polarized (dotted line) light: (a) multipass waveguide geometry of the prepared sample, (b) photoinduced absorption results, (c) FTIR absorption result.

is a phase sensitive technique and signal is detected only when we have additional laser excitation. The PIA data shown in Fig. 4b demonstrated the absorption at $0.8 \mathrm{eV}$ for $p$-polarized light and no absorption for reference $s$-polarized light. Such polarization dependence is firmly attributed to IS transitions.

For transmission measurements we used Fourier transform infrared (FTIR) spectroscopy. The normalized FTIR data for the $1.8 \mathrm{~nm}$ QW sample are shown in Fig. 4c. We made the comparison of $s$ - and $p$-polarized signal and we identified the $\approx 50 \%$ IS absorption for $p$-polarized light at $0.8 \mathrm{eV}$. Taking into account sample size, in this multipass waveguide geometry we have $\approx 1.92 \%$ absorption per one pass. The both PIA and FTIR transmission experiments are consistent with first approximation calculation done for GaN/AIN IS structures [11], where presence of small indium content is neglected.

The results for GaInN/AlInN were compared with the counterpart of data for GaN/AlN IS structures. In spite of some similarities (similar energy of the IS transitions for the same QW width and doping level), the absorption for GaN/AlN structures was weaker due to smaller number of QW repetitions. In addition, the most probably due to already started relaxation process, observed by us IS absorption in GaInN/AlInN structures has multipeak character. The results for PIA and FTIR transmission measurements for GaN/AlN structures were not so reproducible and so consistent as for GaInN/AlInN samples.

\section{Conclusions}

We demonstrated the feasibility of fabricating the forty repetition GaInN/AlInN MQW crack free samples on (0001) GaN substrates. Our IS structures are partially strain compensated to the GaN substrate and exhibit strong 
intersubband absorption at telecommunication wavelengths. For the first time strong IS absorption in GaInN/AlInN sample grown on GaN substrate was observed. The good agreement of PIA and FTIR experiments finally proves the intersubband origin of the absorption. This result is very promising for more sophisticated structures e.g. injector regions in quantum cascade lasers or claddings for waveguided structures.

\section{Acknowledgments}

This work was partially supported by the sixth European Framework Program within the STREP project NITWAVE (contract reference No. 004170) and project SPUB-M-NITWAVE of the Polish Ministry of Scientific Research and Information Technology.

\section{References}

[1] C. Gmachl, H.M. Ng, A.Y. Cho, Appl. Phys Lett. 79, 1590 (2001).

[2] R. Paiella, R. Martini, F. Capasso, C. Gmachl, H.Y. Hwang, D.L. Sivco, J.N. Baillargeon, A.Y. Cho, E.A. Whittaker, H.C. Liu, Appl. Phys Lett. 79, 2526 (2001).

[3] C. Gmachl, H.M. Ng, S. Chu, A.Y. Cho, Appl. Phys Lett. 77, 3722 (2000).

[4] N. Iizuka, K. Kaneko, N. Suzuki, T. Asano, S. Noda, O. Wada, Appl. Phys. Lett. 77, 648 (2000).

[5] H.M. Ng, C. Gmachl, S.N.G. Chu, A.Y. Cho, J. Cryst. Growth 220, 432 (2000).

[6] J. Hamazaki, H. Kunugita, K. Ema, A. Kikuchi, K. Kishino, Phys. Rev. B 71, 165334 (2005).

[7] M. Tchernycheva, L. Nevou, L. Doyennette, F.H. Julien, E. Warde, F. Guillot, E. Monroy, E. Bellet-Amalric, T. Remmele, M. Albrecht, Phys. Rev. B 73, 125347 (2006).

[8] J.D. Heber, C. Gmachl, H.M. Ng, A.Y. Cho, Appl. Phys. Lett. 81, 1237 (2002).

[9] N. Iizuka, K. Kaneko, N. Suzuki, Appl. Phys. Lett. 81, 1803 (2002).

[10] J. Carlin, M. Ilegems, Appl. Phys. Lett. 83, 668 (2003).

[11] G. Cywiński, C. Skierbiszewski, A. Feduniewicz-Żmuda, M. Siekacz, L. Nevou, L. Doyennette, M. Tchernycheva, F.H. Julien, P. Prystawko, M. Kryśko, S. Grzanka, I. Grzegory, A. Presz, J.Z. Domagała, J. Smalc, M. Albrecht, T. Remmele, S. Porowski, J. Vac. Sci. Technol. B 24, 1505 (2006). 